\title{
ON CERTAIN GENERALIZATIONS OF THE SPIRAL-LIKE AND ROBERTSON FUNCTIONS
}

\author{
M. K. AOUF AND H. E. ELATTAR
}

Abstract. Let $S^{\lambda}(\alpha, \beta, A, B)$ denote the class of functions $f(z)=z+\sum_{n=2}^{\infty} a_{n} z^{n}$ which are analytic in the unit $\operatorname{disc} U=\{z:|z|<1\}$ and satisfy the inequality

$$
\left|\frac{\frac{z f^{\prime}(z)}{f(z)}-1}{(B-A) \beta\left(\frac{z f^{\prime}(z)}{f(z)}-1+(1-\alpha) e^{-i \lambda} \cos \lambda\right)+A\left(\frac{z f^{\prime}(z)}{f(z)}-1\right)}\right|<1
$$

for some $\lambda, \alpha, \beta, A, B(|\lambda|<\pi / 2,0 \leq \alpha<1,0<\beta \leq 1,-1 \leq A<B \leq 1$ and $0<B \leq 1)$ and for all $z \in U$. Further $f(z)$ is said to belong to the class $C^{\lambda}(\alpha, \beta, A, B)(|\lambda|<\pi / 2,0 \leq \alpha<1,0<\beta \leq 1,-1 \leq A<B \leq 1$ and $0<B \leq 1)$ if and only if $z f^{\prime}(z) \in S^{\lambda}(\alpha, \beta, A, B)$. In the present paper, the authors give several representation formulas, distortion theorems, and coefficient bounds for functons belonging to these classes. They also obtain the sharp radius of $\gamma$-spiral and starlikeness for the class $S^{\lambda}(\alpha, \beta, A, B)$ and the sharp radius of $\gamma$-convex and convexity for the class $C^{\lambda}(\alpha, \beta, A, B)$.

\section{Introduction}

Let $S$ denote the class of functions

$$
f(z)=z+\sum_{n=2}^{\infty} a_{n} z^{n}
$$

which are analytic in the unit disc $U=\{z:|z|<1\}$. We use $\Omega$ to denote the class of bounded analytic functions $w(z)$ in $U$, satisfying the conditions $w(0)=0$ and $|w(z)|<1$ for $z \in U$.

For $|\lambda|<\frac{\pi}{2}, 0 \leq \alpha<1$ and $-1 \leq A<B \leq 1,0<B \leq 1$, let $S^{\lambda}(\alpha, A, B)$ be the class of those functions $f(z)$ of $S$ for which $\frac{z f^{\prime}(z)}{f(z)}$ is subordinate to $\frac{1+\left[B+(A-B)(1-\alpha) e^{-i \lambda} \cos \lambda\right] z}{1+B z}$.

Received November 19, 1993.

1991 Mathematics Subject Classification. 30C45.

Key words and phrases. Analytic, starlike, convex, coefficient, distortion. 
In other words $f(z) \in S^{\lambda}(\alpha, A, B)$ if and only if there exists a function $w(z) \in \Omega$ such that

$$
\frac{z f^{\prime}(z)}{f(z)}=\frac{1+\left[B+(A-B)(1-\alpha) e^{-i \lambda} \cos \lambda\right] w(z)}{1+B w(z)} .
$$

And the above condition is equivalent to

$$
\left|\frac{\frac{z f^{\prime}(z)}{f(z)}-1}{(B-A)\left(\frac{z f^{\prime}(z)}{f(z)}-1+(1-\alpha) e^{-i \lambda} \cos \lambda\right)+A\left(\frac{z f^{\prime}(z)}{f(z)}-1\right)}\right|<1, z \in U .
$$

The class $S^{\lambda}(\alpha, A, B)$ was introduced by Aouf [4].

Motivated by $[1,14,23]$, we in the present paper, introduce the classes $S^{\lambda}(\alpha, \beta, A, B)$ and $C^{\lambda}(\alpha, \beta, A, B)$, defined as follows:

Definition 1. A function $f(z) \in S$ is in the class $S^{\lambda}(\alpha, \beta, A, B)$ if and only if the inequality

$$
\left|\frac{\frac{z f^{\prime}(z)}{f(z)}-1}{(B-A) \beta\left(\frac{z f^{\prime}(z)}{f(z)}-1+(1-\alpha) e^{-i \lambda} \cos \lambda\right)+A\left(\frac{z f^{\prime}(z)}{f(z)}-1\right)}\right|<1
$$

holds for some $\lambda, \alpha, \beta, A$ and $B\left(|\lambda|<\frac{\pi}{2} ; 0 \leq \alpha<1 ; 0<\beta \leq 1 ;-1 \leq A<B \leq 1\right.$; $0<B \leq 1$ ), and for all $z \in U$.

Definition 2. A function $f(z) \in S$ is in the class $C^{\lambda}(\alpha, \beta, A, B)$ if and only if the inequality

$$
\left|\frac{\frac{z f^{\prime \prime}(z)}{f^{\prime}(z)}}{(B-A) \beta\left(\frac{z f^{\prime \prime}(z)}{f^{\prime}(z)}+(1-\alpha) e^{-i \lambda} \cos \lambda\right)+A \frac{z f^{\prime \prime}(z)}{f^{\prime}(z)}}\right|<1
$$

holds for some $\lambda, \alpha, \beta, A$ and $B\left(|\lambda|<\frac{\pi}{2} ; 0 \leq \alpha<1 ; 0<\beta \leq 1 ;-1 \leq A<B \leq 1\right.$; $0<B \leq 1$ ), and for all $z \in U$.

It follows immediately from Definition 1 and Definition 2 that

$$
f(z) \in C^{\lambda}(\alpha, \beta, A, B) \text { if and only if } z f^{\prime}(z) \in S^{\lambda}(\alpha, \beta, A, B)
$$

We note that $S^{\lambda}(\alpha, \beta,-1,1)=S^{\lambda}(\alpha, \beta)$ is the class of $\lambda$-spiral-like functions of order $\alpha$ and type $\beta\left(|\lambda|<\frac{\pi}{2} ; 0 \leq \alpha<1 ; 0<\beta \leq 1\right)$ which was studied earlier by Mogra and Ahuja [23]. On the other hand, $C^{\lambda}(\alpha, \beta,-1,1)=C^{\lambda}(\alpha, \beta)$ is the class of $\lambda$-Robertson functions of order $\alpha$ and type $\beta\left(|\lambda|<\frac{\pi}{2} ; 0 \leq \alpha<1 ; 0<\beta \leq 1\right)$ which was studied earlier by Ahuja [1].

Furthermore, by specializing the parameters $\lambda, \alpha, \beta, A$ and $B$, we obtain the following subclasses studied earlier by various authors.

(i) $S^{\circ}(\alpha, \beta, A, B)=S^{*}(\alpha, \beta, A, B)$ (Aouf [6]); 
(ii) $S^{\circ}(\alpha, 1, A, B)=S^{*}(\alpha,, A, B)$ (Aouf [5]);

(iii) $S^{\circ}(0,1, A, B)=S^{*}(A, B)$ (Goel and Mehrok [10,11], Janowski [13]);

(iv) $S^{\lambda}(0,1, A, B)=S^{\lambda}(A, B)$ (Dashrath and Shukla [9], Kumar and Shulka [17]);

(v) $S^{\lambda}(\alpha, 1, A, B)=S^{\lambda}(\alpha, A, B)$ (Aouf [2]);

(vi) $S^{\lambda}(\alpha, 1,-1,1)=S^{\lambda}(\alpha)$ (Libera [18], and Patil and Thakare [26]);

(vii) $S^{\lambda}(0,1,-1,1)=S^{\lambda}$ (Špaček [29] and Zamorski [31]);

(viii) $S^{\lambda}\left(0, \frac{2-\cos \lambda}{2},-1,1\right)=H(\lambda)$ (Goel [10]);

(ix) $S^{\lambda}\left(\frac{1-\beta+2 \alpha \beta}{1+\beta}, \frac{1+\beta}{2},-1,1\right)=S_{\alpha, \beta}^{\lambda}$ (Maköwka [20] and Gopalakrishna and Umarani [12]);

(x) $S^{\circ}\left(\frac{1-\beta}{1+\beta}, \frac{1+\beta}{2},-1,1\right)=S(\beta)$ (Padmanabhan [25] and Mogra [21]);

(xi) $S^{\circ}(\alpha, \beta,-1,1)=S^{*}(\alpha, \beta)$ (Juneja and Mogra [14]);

(xii) $S^{\circ}\left(\alpha, \frac{1}{2},-1,1\right)=\bar{S}_{\alpha}$ (Wright [30]);

(xiii) $C^{\lambda}(\alpha, 1,-1,1)=C^{\lambda}(\alpha)$ (Chichra [8] and Sizuk [28]);

(xiv) $C^{\lambda}(1,1,-1,1)=C^{\lambda}$ (Robertson [27], Libera and Ziegler [19], and Bajpai and Mehrok [7]);

(xv) $S^{\lambda}\left(0, \frac{2 M-1}{2 M},-1,1\right)=F_{\lambda, M}$ and $C^{\lambda}\left(0, \frac{2 M-1}{2 M},-1,1\right)=G_{\lambda, M}\left(M>\frac{1}{2}\right)$ (Kulshresha [16]),

and

(xvi) $S^{\lambda}\left(\alpha, \frac{2 M-1}{2 M},-1,1\right)=F_{M}(\lambda, \alpha)$ and $C^{\lambda}\left(\alpha, \frac{2 M-1}{2 M},-1,1\right)=G_{M}(\lambda, \alpha)\left(M>\frac{1}{2}\right)$ (Aouf $[2,3])$.

Note. Although $S^{\lambda}(\alpha, \beta, A, B) \subset S^{\lambda}(\alpha, \beta)$ and $C^{\lambda}(\alpha, \beta, A, B) \subset C^{\lambda}(\alpha, \beta)$, the functions in the class $C^{\lambda}(\alpha, \beta, A, B)$ need not be univalent in $U$, as shown in $[1,27]$.

\section{Representation Formulas}

Let $Q$ denote the class of functions $\varphi(z)$ which are analytic in $U$ and which satisfy $|\varphi(z)| \leq 1$ for all $z \in U$. We require the following lemma.

Lemma. 1. If a function

$$
H(z)=1+\sum_{n=1}^{\infty} d_{n} z^{n}
$$

is analytic in $U$ and satisfies the condition

$$
\left|\frac{H(z)-1}{(B-A) \beta\left(H(z)-1+(1-\alpha) e^{-i \lambda} \cos \lambda\right)+A(H(z)-1)}\right|<1,
$$

for $|\lambda|<\frac{\pi}{2}, 0 \leq \alpha<1,0<\beta \leq 1,-1 \leq A<B \leq 1,0<B \leq 1$, and for all $z \in U$, then

$$
H(z)=\frac{1+\left\{[(B-A) \beta+A]-(B-A) \beta(1-\alpha) e^{-i \lambda} \cos \lambda\right\} z \varphi(z)}{1+[(B-A) \beta+A] z \varphi(z)}
$$


for some $\varphi(z) \in Q$. Conversely, a function $H(z)$ given by (2.2) for some $\varphi(z) \in Q$ is analytic in $U$ and satisfies (2.2) for all $z \in U$.

Proof. The first part of Lemma 1 is obtained immediately by an application of Schwarz's Lemma [24]. The second part follows from the observation that the function

$$
w=\frac{1+\left\{[(B-A) \beta+A]-(B-A) \beta(1-\alpha) e^{-i \lambda} \cos \lambda\right\} z}{1+[(B-A) \beta+A] z}
$$

maps $U$ onto the disc

$$
\left|\frac{1-w}{(B-A) \beta\left(w-1+(1-\alpha) e^{-i \lambda} \cos \lambda\right)+A(w-1)}\right|<1
$$

in the $w$-plane.

Theorem 1. A function $f(z)$, defined by (1.1) and analytic in $U$, is in the class $S^{\lambda}(\alpha, \beta, A, B)$ if and only if

$$
f(z)=z \exp \left\{-(B-A) \beta(1-\alpha) e^{-i \lambda} \cos \lambda \int_{0}^{z} \frac{\varphi(t)}{1+[(B-A) \beta+A] t \varphi(t)} d t\right\} .
$$

for some $\varphi(z) \in Q$.

Proof. First suppose that $f(z) \in S^{\lambda}(\alpha, \beta, A, B)$. Noting that $\frac{z f^{\prime}(z)}{f(z)}$ satisfies the hypothesis of the first part of Lemma 1 , we see that

$$
\frac{z f^{\prime}(z)}{f(z)}=\frac{1+\left\{[(B-A) \beta+A]-(B-A) \beta(1-\alpha) e^{-i \lambda} \cos \lambda\right\} z \varphi(z)}{1+[(B-A) \beta+A] z \varphi(z)}
$$

for some function $\varphi(z) \in Q$. It is easily observed from (2.7) that

$$
\frac{f^{\prime}(z)}{f(z)}-\frac{1}{z}=-\frac{(B-A) \beta(1-\alpha) e^{-i \lambda} \varphi(z) \cos \lambda}{1+[(B-A) \beta+A] z \varphi(z)} .
$$

Upon integrating both sides of (2.8) from 0 to $z$, if we exponentiate the resulting equation, we obtain the representation formula (2.6).

Conversely, if (2.6) holds true, then

$$
\frac{z f^{\prime}(z)}{f(z)}=\frac{1+\left\{[(B-A) \beta+A]-(B-A) \beta(1-\alpha) e^{-i \lambda} \cos \lambda\right\} z \varphi(z)}{1+[(B-A) \beta+A] z \varphi(z)}(z \in U ; \varphi(z) \in Q) .
$$

Now Theorem 1 follows by appealing to the second part of Lemma 1. 
An immediate consequence of Theorem 1, and a representation theorem for functions in $S^{*}(\alpha, \beta, A, B)$ given by Aouf [6] may be shown in the following corollary:

Corollary 1. Let the function $f(z)$ defined by (1.1). Then $f(z) \in S^{\lambda}(\alpha, \beta, A, B)$ if and only if there is a function $f_{1}(z) \in S^{*}(\alpha, \beta, A, B)$ such that

$$
f(z)=z\left[\frac{f_{1}(z)}{z}\right]^{e^{-i \lambda} \cos \lambda} \quad(z \in U) .
$$

In view of the relationship (1.6), it is not difficult to deduce from the above results the following representation formulas for functions belonging to the class $C^{\lambda}(\alpha, \beta, A, B)$ :

Corollary 2. A function $f(z)$ defined by (1.1) is in the class $C^{\lambda}(\alpha, \beta, A, B)$ if and only if its derivative $f^{\prime}(z)$ can be represented as follows:

(i)

$$
f^{\prime}(z)=\left[f_{2}^{\prime}(z)\right]^{e^{-i \lambda} \cos \lambda}
$$

(ii)

for $f_{2}(z) \in C^{\circ}(\alpha, \beta, A, B)=C^{*}(\alpha, \beta, A, B)$

$$
f^{\prime}(z)=\exp \left\{-(B-A) \beta(1-\alpha) e^{-i \lambda} \cos \lambda \int_{0}^{z} \frac{\varphi(t) d t}{1+[(B-A) \beta+A] t \varphi(t)}\right\},
$$

for some function $\varphi(z) \in Q$.

\section{A. Sufficient Condition}

We now establish a sufficient condition for a function to be in each of the classes $S^{\lambda}(\alpha, \beta, A, B)$ and $C^{\lambda}(\alpha, \beta, A, B)$.

Theorem 2. Let the function $f(z)$ defined by (1.1) be analytic in $U$. Then $f(z) \in S^{\lambda}(\alpha, \beta, A, B)$ if, for some $\lambda, \alpha, A$ and $B\left(|\lambda|<\frac{\pi}{2} ; 0 \leq \alpha<1 ;-1 \leq A<B \leq\right.$ 1: $0<B \leq 1$ ),

$$
\begin{gathered}
\sum_{n=2}^{\infty}\left\{n[1-A-(B-A) \beta]-1+\left|[-A-(B-A) \beta]+(B-A) \beta(1-\alpha) e^{-i \lambda} \cos \lambda\right|\right\}\left|a_{n}\right| \\
\leq(B-A) \beta(1-\alpha) \cos \lambda
\end{gathered}
$$

whenever $0<\beta \leq\left(\frac{A}{A-B}\right)$, and

$$
\begin{gathered}
\sum_{n=2}^{\infty}\left\{(n-1)+\left|[(B-A) \beta+A](n-1)+(B-A) \beta(1-\alpha) e^{-i \lambda} \cos \lambda\right|\right\}\left|a_{n}\right| \\
\leq(B-A) \beta(1-\alpha) \cos \lambda,
\end{gathered}
$$


whenever $\left(\frac{A}{A-B}\right) \leq \beta \leq 1$.

Proof. Let $|z|=r<1$. Noting that

$$
\left|z f^{\prime}(z)-f(z)\right|<\sum_{n=2}^{\infty}(n-1)\left|a_{n}\right| r
$$

and

$$
\begin{aligned}
& \left|(B-A) \beta\left[z f^{\prime}(z)-f(z)+(1-\alpha) e^{-i \lambda} f(z) \cos \lambda\right]+A\left[z f^{\prime}(z)-f(z)\right]\right| \\
& >\left\{(B-A) \beta(1-\alpha) \cos \lambda-\sum_{n=2}^{\infty}[-A-(B-A) \beta] n\left|a_{n}\right|\right. \\
& \left.\quad-\sum_{n=2}^{\infty}\left|[-A-(B-A) \beta]+(B-A) \beta(1-\alpha) e^{-i \lambda} \cos \lambda\right|\left|a_{n}\right|\right\} r
\end{aligned}
$$

we see that

$$
\begin{aligned}
& \left|z f^{\prime}(z)-f(z)\right|-\mid(B-A) \beta\left[z f^{\prime}(z)-f(z)+(1-\alpha) e^{-i \lambda} f(z) \cos \lambda\right] \\
+ & A\left[z f^{\prime}(z)-f(z)\right] \mid<\left[\sum_{n=2}^{\infty}\{n[1-A-(B-A) \beta-1+\mid[-A-(B-A) \beta]\right. \\
+ & \left.\left.(B-A) \beta(1-\alpha) e^{-i \lambda} \cos \lambda \mid\right\}\left|a_{n}\right|-(B-A) \beta(1-\alpha) \cos \lambda\right] r,
\end{aligned}
$$

provided that $0<\beta \leq\left(\frac{A}{A-B}\right)$. The right-hand side of (3.5) is non-positive by (3.1), so that $f(z) \in S^{\lambda}(\alpha, \beta, A, B)$ by Definition 1 .

For the second part, we assume that (3.2) holds true for $\left(\frac{A}{A-B}\right) \leq \beta \leq 1$. In this case, we observe that

$$
\begin{aligned}
& \left|(B-A) \beta\left[z f^{\prime}(z)-f(z)+(1-\alpha) e^{-i \lambda} f(z) \cos \lambda\right]+A\left[z f^{\prime}(z)-f(z)\right]\right| \\
> & \left\{(B-A) \beta(1-\alpha) \cos \lambda-\sum_{n=2}^{\infty} \mid[(B-A) \beta+A](n-1)\right. \\
& \left.+(B-A) \beta(1-\alpha) e^{-i \lambda} \cos \lambda|| a_{n} \mid\right\} r .
\end{aligned}
$$

Making using of (3.3), (3.6), and (3.2), we complete the proof of Theorem 2.

Corollary 3. Let the function $f(z)$ defined by (1.1) be analytic in $U$. Then $f(z)$ is in the class $C^{\lambda}(\alpha, \beta, A, B)$ if, for some $\lambda, \alpha, A$ and $B\left(|\lambda|<\frac{\pi}{2} ; 0 \leq \alpha<1\right.$; $-1 \leq A<B \leq 1 ; 0<B \leq 1)$.

$$
\begin{gathered}
\sum_{n=2}^{\infty} n\left\{n[1-A-(B-A) \beta]-1+\left|[-A-(B-A) \beta]+(B-A) \beta(1-\alpha) e^{-i \lambda} \cos \lambda\right|\right\}\left|a_{n}\right| \\
\leq(B-A) \beta(1-\alpha) \cos \lambda
\end{gathered}
$$


whenever $0<\beta \leq\left(\frac{A}{A-B}\right)$, and

$$
\begin{aligned}
\sum_{n=2}^{\infty} n\{(n-1)+ & \left.\left|[(B-A) \beta+A](n-1)+(B-A) \beta(1-\alpha) e^{-i \lambda} \cos \lambda\right|\right\}\left|a_{n}\right| \\
\leq & (B-A) \beta(1-\alpha) \cos \lambda,
\end{aligned}
$$

whenever $\left(\frac{A}{A-B}\right) \leq \beta \leq 1$.

Proof. Since

$$
z f^{\prime}(z)=z+\sum_{n=2}^{\infty} n a_{n} z^{n}
$$

by replacing $a_{n}$ by $n a_{n}$ in Theorem 2, we immediately have Corollary 3 in view of the equivalence relation (1.6).

For various choices of the parameters involved in Theorem 2 and Corollary 3 , we can obtain the corresponding results for functions belonging to the numerous simpler classes described in Section 1.

\section{Ditortion Theorems} then

Theorem 3. If a function $f(z)$ defined by (1.1) is in the class $S^{\lambda}(\alpha, \beta, A, B)$,

$$
|f(z)| \leq r\left[\frac{(1+[(B-A) \beta+A] r)^{(1-\cos \lambda)}}{(1-[(B-A) \beta+A] r)^{(1+\cos \lambda)}}\right]^{\frac{(B-A) \beta(1-\alpha) \cos \lambda}{2((B-A) \beta+A]}}
$$

and

$$
|f(z)| \geq r\left[\frac{(1-[(B-A) \beta+A] r)^{(1-\cos \lambda)}}{(1+[(B-A) \beta+A] r)^{(1+\cos \lambda)}}\right]^{\frac{(B-A) \beta(1-\alpha) \cos \lambda}{2((B-A) \beta+A]}}
$$

for $|z|=r(0<r<1),|\lambda|<\frac{\pi}{2}, 0 \leq \alpha<1,0<\beta \leq 1, \beta \neq\left(\frac{A}{A-B}\right),-1 \leq A<B \leq 1$, and $0<B \leq 1$; and

$$
|f(z)| \leq r \exp [-A(1-\alpha) r \cos \lambda]
$$

and

$$
|f(z)| \geq r \exp [A(1-\alpha) r \cos \lambda]
$$

for $|z|=r(0<r<1),|\lambda|<\frac{\pi}{2}, 0 \leq \alpha<1, \beta=\left(\frac{A}{A-B}\right),-1 \leq A<B \leq 1$, and $0<B \leq 1$.

All these estimates are sharp for all admissible values of $\lambda, \alpha, \beta, A$ and $B$.

Proof. Since $f(z) \in S^{\lambda}(\alpha, \beta, A, B)$, the condition (1.4) coupled with an application of Schwarz's Lemma [24] implies that

$$
\left|\frac{z f^{\prime}(z)}{f(z)}-\xi\right|<R
$$


where

$$
\begin{aligned}
\xi= & {\left[1-[(B-A) \beta+A]\left\{[(B-A) \beta+A]-(B-A) \beta(1-\alpha) \cos ^{2} \lambda\right\} r^{2}\right.} \\
& \left.-i 2^{-1}(B-A) \beta[(B-A) \beta+A](1-\alpha) r^{2} \sin 2 \lambda\right]\left[1-[(B-A) \beta+A]^{2} r^{2}\right]^{-1}
\end{aligned}
$$

and

$$
R=\frac{(B-A) \beta(1-\alpha) r \cos \lambda}{1-[(B-A) \beta+A]^{2} r^{2}} \quad(|z|=r)
$$

Hence we obtain

$\frac{1-(B-A) \beta(1-\alpha) r \cos \lambda+[(B-A) \beta+A]\left\{(B-A) \beta(1-\alpha) \cos ^{2} \lambda-[(B-A) \beta+A]\right\} r^{2}}{1-[(B-A) \beta+A]^{2} r^{2}}$

$\leq \operatorname{Re}\left\{\frac{z f^{\prime}(z)}{f(z)}\right\} \leq$

$\frac{1+(B-A) \beta(1-\alpha) r \cos \lambda+[(B-A) \beta+A]\left\{(B-A) \beta(1-\alpha) \cos ^{2} \lambda-[(B-A) \beta+A]\right\} r^{2}}{1-[(B-A) \beta+A]^{2} r^{2}}$.

Observing that

$$
\begin{aligned}
\log \left(\left|\frac{f(z)}{z}\right|\right) & =\operatorname{Re}\left(\log \frac{f(z)}{z}\right)=\operatorname{Re}\left[\int_{0}^{z}\left(\frac{f^{\prime}(s)}{f(s)}-\frac{1}{s}\right) d s\right] \\
& =\int_{0}^{r} \frac{1}{t} \operatorname{Re}\left[t e^{i \theta} \frac{f^{\prime}\left(t e^{i \theta}\right)}{f\left(t e^{i \theta}\right)}-1\right] d t
\end{aligned}
$$

and applying (4.5), we find that

$$
\log \left(\left|\frac{f(z)}{z}\right|\right) \leq[(B-A) \beta(1-\alpha) \cos \lambda] \int_{0}^{r} \frac{1+[(B-A) \beta+A] t \cos \lambda}{1-[(B-A) \beta+A]^{2} t^{2}} d t
$$

Now suppose that $|\lambda|<\frac{\pi}{2}, 0 \leq \alpha<1, \beta \neq\left(\frac{A}{A-B}\right),-1 \leq A<B \leq 1$, and $0<B \leq 1$. Then (4.6) yields

$$
\log \left(\left|\frac{f(z)}{z}\right|\right) \leq \frac{(B-A) \beta(1-\alpha) \cos \lambda}{2[(B-A) \beta+A]} \log \left\{\frac{(1+[(B-A) \beta+A] r)^{(1-\cos \lambda)}}{(1-[(B-A) \beta+A] r)^{(1+\cos \lambda)}}\right\}
$$

which leads us to (4.1). For the case when $|\lambda|<\frac{\pi}{2}, 0 \leq \alpha<1, \beta=\left(\frac{A}{A-B}\right),-1 \leq A<$ $B \leq 1$, and $0<B \leq 1,(4.6)$ immediately gives (4.3).

In view of the fact that

$$
\begin{aligned}
\log \left(\left|\frac{f(z)}{z}\right|\right) & =\operatorname{Re}\left(\log \frac{f(z)}{z}\right)=\int_{0}^{r} \operatorname{Re}\left\{\frac{\partial}{\partial t}\left[\log \frac{f(t)}{t}\right]\right\} d t \\
& =\int_{0}^{r} \frac{1}{t} \operatorname{Re}\left\{\frac{t f^{\prime}(t)}{f(t)}-1\right\} d t
\end{aligned}
$$


and with the aid of (4.5), we may write

$$
\log \left(\left|\frac{f(z)}{z}\right|\right) \geq-[(B-A) \beta(1-\alpha) \cos \lambda] \int_{0}^{r} \frac{1-[(B-A) \beta+A] t \cos \lambda}{1-[(B-A) \beta+A]^{2} t^{2}} d t .
$$

If $\beta \neq\left(\frac{A}{A-B}\right)$, then (4.2) follows upon evaluating the integeral in (4.7). If, on the other hand, $\beta=\left(\frac{A}{A-B}\right)$, then we immediately get (4.4) from (4.7).

The extermal function for all of the inequalities is given by

$$
f(z)= \begin{cases}z\left\{1-[(B-A) \beta+A] z e^{i \theta}\right\}^{\frac{-\left[(B-A) \beta(1-\alpha) e^{-i \lambda} \cos \lambda\right.}{[(B-A) \beta+A]}}, & \beta \neq\left(\frac{A}{A-B}\right) \\ z \exp \left[-A(1-\alpha) z e^{i(\theta-\lambda)} \cos \lambda\right], & \beta=\left(\frac{A}{A-B}\right)\end{cases}
$$

where $|\lambda|<\frac{\pi}{2}, 0 \leq \alpha<1,-1 \leq A<B \leq 1,0<B \leq 1$, and $\theta(0 \leq \theta \leq 2 \pi)$ is determined by

$$
\tan \left(\frac{\theta}{2}\right)=\left\{\frac{1-[(B-A) \beta+A] r}{1+[(B-A) \beta+A] r}\right\} \cot \left(\frac{\pi}{2}-\frac{\lambda}{2}\right)
$$

for the equality in (4.1) and (4.3) and by the equation

$$
\tan \left(\frac{\theta}{2}\right)=\left\{\frac{1-[(B-A) \beta+A] r}{1+[(B-A) \beta+A] r}\right\} \cot \left(-\frac{\lambda}{2}\right)
$$

for the equality in (4.2) and (4.4). then

Corollary 4. If a function $f(z)$ defined by (1.1) is in the class $C^{\lambda}(\alpha, \beta, A, B)$,

$$
\left|f^{\prime}(z)\right| \leq\left[\frac{(1+[(B-A) \beta+A] r)^{(1-\cos \lambda)}}{1-[(B-A) \beta+A] r)^{(1+\cos \lambda)}}\right]^{\frac{(B-A) \beta(1-\alpha) \cos \lambda}{2[(B-A) \beta+A]}}
$$

and

$$
\left|f^{\prime}(z)\right| \geq\left[\frac{(1-[(B-A) \beta+A] r)^{(1-\cos \lambda)}}{1+[(B-A) \beta+A] r)^{(1+\cos \lambda)}}\right]^{\frac{(B-A) \beta(1-\alpha) \cos \lambda}{2[(B-A) \beta+A]}}
$$

for $|z|=r<1,|\lambda|<\frac{\pi}{2}, 0 \leq \alpha<1,0<\beta \leq 1, \beta \neq\left(\frac{A}{A-B}\right),-1 \leq A<B \leq 1$, and $0<B \leq 1$; and

$$
\left|f^{\prime}(z)\right| \leq \exp [-A(1-\alpha) r \cos \lambda]
$$

and

$$
\left|f^{\prime}(z)\right| \geq \exp [A(1-\alpha) r \cdot \cos \lambda]
$$

for $|z|=r<1,|\lambda|<\frac{\pi}{2}, 0 \leq \alpha<1, \beta=\left(\frac{A}{A-B}\right),-1 \leq A<B \leq 1$, and $0<B \leq 1$.

The function $f(z)$ given by

$$
f^{\prime}(z)= \begin{cases}\left\{1-[(B-A) \beta+A] z e^{i \theta}\right\}^{\frac{-\left[(B-A) \beta(1-\alpha) e^{-i \lambda} \cos \lambda\right.}{[(B-A) \beta+A]}}, & \beta \neq\left(\frac{A}{A-B}\right) \\ \exp \left[-A(1-\alpha) z e^{i(\theta-\lambda)} \cos \lambda\right], & \beta=\left(\frac{A}{A-B}\right)\end{cases}
$$


provides equality in (4.11) and (4.13) when $\theta$ is given by Equation (4.9). The function $f(z)$ given by (4.15) also provides equality in (4.12) and (4.14) when $\theta$ is given by Equation (4.10).

For various choices of the parameters in Theorem 3 and Corollary 4, the corresponding known or new results can be deduced for functions in the classes studied earlieir in the literature.

\section{Coefficient Bounds}

We shall require the following two lemmas in our investigation.

Lemma 2 [24]. Let the function $w(z)$ defined by

$$
w(z)=\sum_{n=1}^{\infty} c_{n} z^{n}
$$

be in the class $\Omega$. Then

$$
\left|c_{1}\right| \leq 1
$$

and

$$
\left|c_{2}\right| \leq 1-\left|c_{1}\right|^{2}
$$

Lemma $3[15]$. Let the function $w(z)$ defined by (5.1) be in the class $\Omega$. Then

$$
\left|c_{2}-\nu c_{1}^{2}\right| \leq \max \{1,|\nu|\}
$$

for any complex number $\nu$. Equality in (5.4) may be attained with the functions $w(z)=z^{2}$ and $w(z)=z$ for $|\nu|<1$ and $|\nu| \geq 1$, respectively.

Theorem 4. If a function $f(z)$ defined by (1.1) is in the class $S^{\lambda}(\alpha, \beta, A, B)$, $\beta \neq\left(\frac{A}{A-B}\right)$, then

(a) for any real number $\mu$, we have

$$
\left|a_{3}-\mu a_{2}^{2}\right| \leq\left\{\begin{array}{l}
\frac{(B-A) \beta(1-\alpha) \cos \lambda}{2}[\cos \lambda\{(B-A) \beta(1-\alpha)(1-2 \mu) \\
+[(B-A) \beta+A]\}+|[(B-A) \beta+A] \sin \lambda|], \\
\quad \text { if } \mu \leq \frac{-1+A+(B-A) \beta(2-\alpha)}{2(B-A) \beta(1-\alpha)}, \\
\frac{(B-A) \beta(1-\alpha) \cos \lambda}{2}[\cos \lambda+|[(B-A) \beta+A] \sin \lambda|], \\
\text { if } \frac{-1+A+(B-A) \beta(2-\alpha)}{2(B-A) \beta(1-\alpha)} \leq \mu \leq \frac{1+A+(B-A) \beta(2-\alpha)}{2(B-A) \beta(1-\alpha)}, \\
\frac{(B-A) \beta(1-\alpha) \cos \lambda}{2}[\cos \lambda\{(B-A) \beta(1-\alpha)(2 \mu-1) \\
-[(B-A) \beta+A]\}+|[(B-A) \beta+A] \sin \lambda|], \\
\quad \text { if } \mu \geq \frac{1+A+(B-A) \beta(2-\alpha)}{2(B-A) \beta(1-\alpha)},
\end{array}\right.
$$


and

(b) for any complex number $\mu$, we obtain

$$
\begin{aligned}
\left|a_{3}-\mu a_{2}^{2}\right| \leq & \frac{(B-A) \beta(1-\alpha) \cos \lambda}{2} \max \{1, \mid(B-A) \beta(1-\alpha)(2 \mu-1) \cos \lambda \\
& \left.-[(B-A) \beta+A] e^{i \lambda} \mid\right\} .
\end{aligned}
$$

This result is sharp for each $\mu$ either real or complex.

Proof. Since $f(z) \in S^{\lambda}(\alpha, \beta, A, B),(2.7)$ gives

$$
\frac{z f^{\prime}(z)}{f(z)}=\frac{1+\left\{[(B-A) \beta+A]-(B-A) \beta(1-\alpha) e^{-i \lambda} \cos \lambda\right\} w(z)}{1+[(B-A) \beta+A] w(z)},
$$

where $w(z)$ defined by (5.1) is in the class $\Omega$. Rewriting (5.7) in the form

$$
w(z)=\frac{z f^{\prime}(z)-f(z)}{\left\{[(B-A) \beta+A]-(B-A) \beta(1-\alpha) e^{-i \lambda} \cos \lambda\right\} f(z)-[(B-A) \beta+A] z f^{\prime}(z)}
$$

and applying the definition (1.1), it can be shown that

$$
\begin{aligned}
w(z)= & -\frac{1}{(B-A) \beta(1-\alpha) e^{-i \lambda} \cos \lambda} . \\
& \cdot\left[a_{2} z+\left\{2 a_{3}-\frac{[(B-A) \beta+A]+(B-A) \beta(1-\alpha) e^{-i \lambda} \cos \lambda}{(B-A) \beta(1-\alpha) e^{-i \lambda} \cos \lambda} a_{2}^{2}\right\} z^{2}+\cdots\right] .
\end{aligned}
$$

Now compare the coefficients of $z$ and $z^{2}$ on both sides of (5.9), using the definition (5.1). We thus obtain

$$
c_{1}=-\frac{e^{i \lambda} \sec \lambda}{(B-A) \beta(1-\alpha)} a_{2}
$$

and

$$
\begin{aligned}
c_{2}= & -\frac{2 e^{i \lambda} \sec \lambda}{(B-A) \beta(1-\alpha)} a_{3}+\frac{e^{i \lambda} \sec \lambda}{(B-A)^{2} \beta^{2}(1-\alpha)^{2}}\{(B-A) \beta(1-\alpha) \\
& \left.+[(B-A) \beta+A] e^{i \lambda} \sec \lambda\right\} a_{2}^{2} .
\end{aligned}
$$

Consequently, we have

$$
a_{2}=-\frac{(B-A) \beta(1-\alpha)}{\sec \lambda} c_{1} e^{-i \lambda}
$$

and

$$
\begin{aligned}
a_{3}= & -\frac{(B-A) \beta(1-\alpha)}{2 \sec \lambda} c_{2} e^{-i \lambda} \\
& +\left[\frac{(B-A) \beta(1-\alpha)+[(B-A) \beta+A] e^{i \lambda} \sec \lambda}{2(B-A) \beta(1-\alpha)}\right] a_{2}^{2} .
\end{aligned}
$$


Using (5.12) and (5.13), we get

$$
\begin{aligned}
& a_{3}-\mu a_{2}^{2}=-\frac{(B-A) \beta(1-\alpha)}{2 e^{i \lambda} \sec \lambda} c_{2} \\
& +\left[\frac{(B-A) \beta(1-\alpha)+[(B-A) \beta+A] e^{i \lambda} \sec \lambda}{2(B-A) \beta(1-\alpha)}-\mu\right] \frac{(B-A)^{2} \beta^{2}(1-\alpha)^{2}}{e^{2 i \lambda} \sec ^{2} \lambda} c_{1}^{2} .
\end{aligned}
$$

Thus taking modulus of both sides of (5.14), we are led to

$$
\begin{aligned}
\left|a_{3}-\mu a_{2}^{2}\right|= & \frac{(B-A) \beta(1-\alpha)}{2 \sec \lambda} . \\
& \cdot\left|c_{2}-\left\{-\frac{(B-A) \beta(1-\alpha)}{e^{i \lambda} \sec \lambda}(2 \mu-1)+[(B-A) \beta+A]\right\} c_{1}^{2}\right| .
\end{aligned}
$$

(a) When $\mu$ is real.

For real $\mu,(5.15)$ becomes

$$
\begin{aligned}
\left|a_{3}-\mu a_{2}^{2}\right| \leq & \frac{(B-A) \beta(1-\alpha)}{2 \sec \lambda} . \\
& \cdot\left\{\left|c_{2}\right|+\left|(B-A) \beta(1-\alpha)(2 \mu-1) \cos \lambda-[(B-A) \beta+A] e^{i \lambda}\right|\left|c_{1}\right|^{2}\right\} .
\end{aligned}
$$

Applying Lemma 2 for $\left|c_{2}\right|$ in (5.16) we obtain

$$
\begin{aligned}
\left|a_{3}-\mu a_{2}^{2}\right| \leq & \frac{(B-A) \beta(1-\alpha) \cos \lambda}{2}\{1+[\mid(B-A) \beta(1-\alpha)(2 \mu-1) \cos \lambda \\
& \left.\left.-[(B-A) \beta+A] e^{i \lambda} \mid-1\right]\left|c_{1}\right|^{2}\right\} .
\end{aligned}
$$

Again using Lemma 2 for $\left|c_{1}\right|$ in (5.17) we are led to

$$
\begin{aligned}
\left|a_{3}-\mu a_{2}^{2}\right| \leq & \frac{(B-A) \beta(1-\alpha) \cos \lambda}{2}\{\mid(B-A) \beta(1-\alpha)(2 \mu-1) \cos \lambda \\
& \left.-[(B-A) \beta+A] e^{i \lambda} \mid\right\} \\
\leq & \frac{(B-A) \beta(1-\alpha) \cos \lambda}{2}\{\cos \lambda \mid(B-A) \beta(1-\alpha)(2 \mu-1) \\
& -[(B-A) \beta+A]|+|[(B-A) \beta+A] \sin \lambda \mid\} .
\end{aligned}
$$

Thus from (5.18) with simple computations we obtain the results of (5.5) stated in (a) of Theorem 4 for various values of real $\mu$.

(b) When $\mu$ is a Complex Number.

For any complex number $\mu$ (5.15) may be written as

$$
\begin{aligned}
\left|a_{3}-\mu a_{2}^{2}\right| \leq & \frac{(B-A) \beta(1-\alpha) \cos \lambda}{2} . \\
& \cdot\left|c_{2}-\left\{\frac{[(B-A) \beta+A] e^{i \lambda} \sec \lambda-(B-A) \beta(1-\alpha)(2 \mu-1)}{e^{i \lambda} \sec \lambda}\right\} c_{1}^{2}\right| .
\end{aligned}
$$


Applying Lemma 3 in (5.19) we get

$$
\begin{aligned}
\left|a_{3}-\mu a_{2}^{2}\right| \leq & \frac{(B-A) \beta(1-\alpha) \cos \lambda}{2} \max \{1, \\
& \left.\left|(B-A) \beta(1-\alpha)(2 \mu-1) \cos \lambda-[(B-A) \beta+A] e^{i \lambda}\right|\right\}
\end{aligned}
$$

which is (5.6) in (b) of Theorem 4.

Finally, the assertions (5.5) and (5.6) of Theorem 4 are sharp in view of the fact that the assertion (5.4) of Lemma 3 is sharp.

In a similar way we can prove:

Theorem 5. If a function $f(z)$ defined by (1.1) is in the class $C^{\lambda}(\alpha, \beta, A, B)$, $\beta \neq=\left(\frac{A}{A-B}\right)$, then

(a) for any real number $\mu$, we have

$$
\left|a_{3}-\mu a_{2}^{2}\right| \leq\left\{\begin{array}{l}
\frac{(B-A) \beta(1-\alpha) \cos \lambda}{6}\left[\operatorname { c o s } \lambda \left\{(B-A) \beta(1-\alpha)\left(1-\frac{3}{2} \mu\right)\right.\right. \\
+[(B-A) \beta+A]\}+|[(B-A) \beta+A] \sin \lambda|] \\
\quad \text { if } \mu \leq \frac{2[-1+A+(B-A) \beta(2-\alpha)]}{3(B-A) \beta(1-\alpha)} \\
\frac{(B-A) \beta(1-\alpha) \cos \lambda}{6}[\cos \lambda+|[(B-A) \beta+A] \sin \lambda|] \\
\text { if } \frac{2[-1+A+(B-A) \beta(2-\alpha)]}{3(B-A) \beta(1-\alpha)} \leq \mu \leq \frac{2[1+A+(B-A) \beta(2-\alpha)]}{3(B-A) \beta(1-\alpha)} \\
\frac{(B-A) \beta(1-\alpha) \cos \lambda}{6}\left[\operatorname { c o s } \lambda \left\{(B-A) \beta(1-\alpha)\left(\frac{3}{2} \mu-1\right)\right.\right. \\
-[(B-A) \beta+A]\}+|[(B-A) \beta+A] \sin \lambda|] \\
\text { if } \mu \geq \frac{2[1+A+(B-A) \beta(2-\alpha)]}{3(B-A) \beta(1-\alpha)}
\end{array}\right.
$$

and

(b) for any complex number $\mu$, we obtain

$$
\begin{aligned}
\left|a_{3}-\mu a_{2}^{2}\right| \leq & \frac{(B-A) \beta(1-\alpha) \cos \lambda}{6} \max \left\{1, \mid(B-A) \beta(1-\alpha)\left(\frac{3}{2} \mu-1\right) \cos \lambda\right. \\
& \left.-[(B-A) \beta+A] e^{i \lambda} \mid\right\} .
\end{aligned}
$$

This result is sharp for each $\mu$ either real or complex.

Theorem 6. Let the function $f(z)$ defined by (1.1) be in the class $S^{\lambda}(\alpha, \beta, A, B)$.

(a) If

$$
\begin{gathered}
\beta(1-\alpha)(k-\alpha) \cos ^{2} \lambda>\frac{(k-1)}{(B-A)^{2} \beta}\left\{(k-1)\left(1-A^{2}\right)-(B-A) \beta[(B-A) \beta+2 A] .\right. \\
\left.\cdot\left[(1-\alpha) \cos ^{2} \lambda+k-1\right]\right\}
\end{gathered}
$$


let

$$
N=\left[\frac{\beta(1-\alpha)(k-\alpha) \cos ^{2} \lambda}{\frac{(k-1)}{(B-A)^{2} \beta}\left\{(k-1)\left(1-A^{2}\right)-(B-A) \beta[(B-A) \beta+2 A]\left[(1-\alpha) \cos ^{2} \lambda+k-1\right]\right\}}\right],
$$

$k=2,3 \ldots, n-1$. Then

$$
\begin{gathered}
\left|a_{n}\right| \leq \frac{1}{(n-1) !} \prod_{k=2}^{n}\left|[(B-A) \beta+A](k-2)+(B-A) \beta(1-\alpha) e^{-i \lambda} \cos \lambda\right| \\
n>N+2 .
\end{gathered}
$$

for $n=2,3 \ldots, N+2 ;$ and

$$
\begin{gathered}
\left|a_{n}\right| \leq \frac{1}{(N+1) !(n-1)} \prod_{k=2}^{N+3}\left|[(B-A) \beta+A](k-2)+(B-A) \beta(1-\alpha) e^{-i \lambda} \cos \lambda\right| \\
n>N+2 .
\end{gathered}
$$

(b) If, on the other hand,

$$
\begin{gathered}
\beta(1-\alpha)(k-\alpha) \cos ^{2} \lambda \leq \frac{(k-1)}{(B-A)^{2} \beta}\left\{(k-1)\left(1-A^{2}\right)-(B-A) \beta[(B-A) \beta+2 A] .\right. \\
\left.\cdot\left[(1-\alpha) \cos ^{2} \lambda+k-1\right]\right\}
\end{gathered}
$$

then

$$
\left|a_{n}\right| \leq \frac{(B-A) \beta(1-\alpha) \cos \lambda}{(n-1)} \text { for } n \geq 2 .
$$

The bounds in (5.23) and (5.26) are sharp for all admissible $\alpha, \beta, \lambda, A, B$, and for each $n$.

Proof. Since $f(z) \in S^{\lambda}(\alpha, \beta, A, B)$, (5.7) gives

$$
\begin{gathered}
{\left[[(B-A) \beta+A] z f^{\prime}(z)-\left\{[(B-A) \beta+A]-(B-A) \beta(1-\alpha) e^{-i \lambda} \cos \lambda\right\} f(z)\right] w(z)} \\
=f(z)-z f^{\prime}(z), \quad w(z) \in \Omega
\end{gathered}
$$

Rewriting (5.27) in the form:

$$
\begin{gathered}
{\left[[(B-A) \beta+A]\left[z+\sum_{k=2}^{\infty} k a_{k} z^{k}\right]-\left\{[(B-A) \beta+A]-(B-A) \beta(1-\alpha) e^{-i \lambda} \cos \lambda\right\} .\right.} \\
\left.\cdot\left[z+\sum_{k=2}^{\infty} a_{k} z^{k}\right]\right] w(z)=\sum_{k=2}^{\infty}(1-k) a_{k} z^{k}
\end{gathered}
$$


or, equivalently,

$$
\begin{aligned}
& {\left[(B-A) \beta(1-\alpha) z e^{-i \lambda} \cos \lambda+\sum_{k=2}^{\infty}\{[(B-A) \beta+A](k-1)\right.} \\
& \left.\left.\quad+(B-A) \beta(1-\alpha) e^{-i \lambda} \cos \lambda\right\} a_{k} z^{k}\right] w(z) \\
& =\sum_{k=2}^{\infty}(1-k) a_{k} z^{k}
\end{aligned}
$$

where $w(z)$ is given, as before, by (5.1).

Equating corresponding coefficients on both sides of (5.28) we observe that the coefficients $a_{n}$ on the right-hand side depends only on the coefficients $a_{2}, a_{3}, \ldots, a_{n-1}(n \geq 2)$ occuring on the left-hand side. Hence for $n \geq 2$, it follows from (5.28) that

$$
\begin{aligned}
& {\left[(B-A) \beta(1-\alpha) z e^{-i \lambda} \cos \lambda+\sum_{k=2}^{n-1}\{[(B-A) \beta+A](k-1)\right.} \\
& \left.\left.\quad+(B-A) \beta(1-\alpha) e^{-i \lambda} \cos \lambda\right\} a_{k} z^{k}\right] w(z) \\
& =\sum_{k=2}^{n}(1-k) a_{k} z^{k}+\sum_{k=n+1}^{\infty} b_{k} z^{k},
\end{aligned}
$$

where $\sum_{k=n+1}^{\infty} b_{k} z^{k}$ converges in $U$. Writing $z=r e^{i \theta}$, integrating from 0 to $2 \pi$ and using the bound $|w(z)| \leq|z|$ for $z \in U,(5.29)$ gives

$$
\begin{gathered}
\frac{1}{2 \pi} \int_{0}^{2 \pi} \mid(B-A) \beta(1-\alpha) r e^{i \theta} e^{-i \lambda} \cos \lambda+\sum_{k=2}^{n-1}\{[(B-A) \beta+A](k-1) \\
\left.+(B-A) \beta(1-\alpha) e^{-i \lambda} \cos \lambda\right\}\left.a_{k} r^{k} e^{i k \theta}\right|^{2} d \theta \\
\geq \frac{1}{2 \pi} \int_{0}^{2 \pi}\left|\sum_{k=2}^{n}(1-k) a_{k} r^{k} e^{i k \theta}+\sum_{k=n+1}^{n} b_{k} r^{k} e^{i k \theta}\right|^{2} d \theta
\end{gathered}
$$

which by Parseval's identity [24] is equivalent to

$$
\begin{aligned}
(B-A)^{2} \beta^{2}(1-\alpha)^{2} r^{2} \cos ^{2} \lambda & +\sum_{k=2}^{n-1} \mid[(B-A) \beta+A](k-1) \\
& +\left.(B-A) \beta(1-\alpha) e^{-i \lambda} \cos \lambda\right|^{2}\left|a_{k}\right|^{2} \\
\geq & \sum_{k=2}^{n}(1-k)^{2}\left|a_{k}\right|^{2} r^{2 k}+\sum_{k=n+1}^{\infty}\left|b_{k}\right|^{2} r^{2 k} .
\end{aligned}
$$

Since the infinite series in (5.30) is non-negative for $0<r<1$, we have as $r \rightarrow 1$

$$
\begin{aligned}
& (n-1)^{2}\left|a_{n}\right|^{2} \leq(B-A)^{2} \beta^{2}(1-\alpha)^{2} \cos ^{2} \lambda+\sum_{k=2}^{n-1}\{\mid[(B-A) \beta+A][(k-1) \\
& \left.+\left.(B-A) \beta(1-\alpha) e^{-i \lambda} \cos \lambda\right|^{2}-(k-1)^{2}\right\}\left|a_{k}\right|^{2}, \quad n \geq 2 .
\end{aligned}
$$


The following two cases will now arise:

(a) Let the inequality (5.21) hold. Suppose also that $n \leq N+2$ in (5.31), where $N$ is given by (5.22). Then, for $n=2,(5.31)$ immediately yields

$$
\left|a_{2}\right| \leq(B-A) \beta(1-\alpha) \cos \lambda,
$$

which proves (5.23) for $n=2$. We establish (5.23) for $n \leq N+2$, from (5.31), by mathematical induction.

Fix $n, n \geq 3$, and suppose that (5.23) holds for $k=2,3 \ldots, n-1$. Then it follows from (5.31) that

$$
\begin{aligned}
& \left|a_{n}\right|^{2} \leq \frac{1}{(n-1)^{2}}\left\{(B-A)^{2} \beta^{2}(1-\alpha)^{2} \cos ^{2} \lambda+\sum_{k=2}^{n-1}[\mid[(B-A) \beta+A](k-1)\right. \\
& \left.\left.\quad+\left.M\right|^{2}-(k-1)^{2}\right] \frac{1}{((k-1) !)^{2}} \prod_{j=2}^{k}|[(B-A) \beta+A](j-2)+M|^{2}\right\},
\end{aligned}
$$

where $M=(B-A) \beta(1-\alpha) e^{-i \lambda} \cos \lambda$.

We must show that the square of the right-hand side of (5.23) is equal to the right-hand side of (5.33); that is,

$$
\begin{aligned}
& \frac{1}{((m-1) !)^{2}} \prod_{j=2}^{m}|[(B-A) \beta+A](j-2)+M|^{2} \\
= & \frac{1}{(m-1)^{2}}\left\{(B-A)^{2} \beta^{2}(1-\alpha)^{2} \cos ^{2} \lambda+\sum_{k=2}^{m-1}\left[|[(B-A) \beta+A](k-1)+M|^{2}\right.\right. \\
& \left.\left.-(k-1)^{2}\right] \cdot \frac{1}{((k-1) !)^{2}} \prod_{j=2}^{k}|[(B-A) \beta+A](j-2)+M|^{2}\right\}
\end{aligned}
$$

for $m=3,4, \ldots$. A brief calculation verifies (5.34) for $m=3$ and proves (5.23) for $n=3$. Assume that (3.23) is valid for all $m, 3<m \leq n-1$; then (5.31) and (5.33) give

$$
\begin{aligned}
\left|a_{n}\right|^{2} \leq & \frac{1}{(n-1)^{2}}\left\{(B-A)^{2} \beta^{2}(1-\alpha)^{2} \cos ^{2} \lambda\right. \\
+ & \sum_{k=2}^{n-2}\left[|[(B-A) \beta+A](k-1)+M|^{2}-(k-1)^{2}\right] . \\
& \cdot \frac{1}{((k-1) !)^{2}} \prod_{j=2}^{k}|[(B-A) \beta+A](j-2)+M|^{2} \\
+ & {\left[|[(B-A) \beta+A](n-2)+M|^{2}-(n-2)^{2}\right] . } \\
& \left.\cdot \frac{1}{((n-2) !)^{2}} \prod_{j=2}^{n-1}|[(B-A) \beta+A](j-2)+M|^{2}\right\}
\end{aligned}
$$




$$
\begin{aligned}
= & \frac{(n-2)^{2}}{(n-1)^{2}}\left[\frac { 1 } { ( n - 2 ) ^ { 2 } } \left\{(B-A)^{2} \beta^{2}(1-\alpha)^{2} \cos ^{2} \lambda\right.\right. \\
& +\sum_{k=2}^{n-1}\left[|[(B-A) \beta+A](k-1)+M|^{2}-(k-1)^{2}\right] . \\
& \left.\cdot \frac{1}{((k-1) !)^{2}} \prod_{j=2}^{k}|[(B-A) \beta+A](j-2)+M|^{2}\right\} \\
& +\frac{1}{(n-2)^{2}}\left\{|[(B-A) \beta+A](n-2)+M|^{2}-(n-2)^{2}\right\} . \\
& \left.\cdot \frac{1}{((n-2) !)^{2}} \prod_{j=2}^{n-1}|[(B-A) \beta+A](j-2)+M|^{2}\right]
\end{aligned}
$$

or

$$
\begin{aligned}
\left|a_{n}\right|^{2} \leq & \frac{(n-2)^{2}}{(n-1)^{2}}\left[\frac{1}{((n-2) !)^{2}} \prod_{j=2}^{n-1}|[(B-A) \beta+A](j-2)+M|^{2}\right] \\
& +\frac{1}{((n-1) !)^{2}} \cdot\left[|[(B-A) \beta+A](n-2)+M|^{2}-(n-2)^{2}\right] . \\
& \cdot\left[\prod_{j=2}^{n-1}|[(B-A) \beta+A](j-2)+M|^{2}\right] \\
= & \frac{1}{((n-1) !)^{2}}\left[|[(B-A) \beta+A](n-2)+M|^{2}\right] . \\
& \cdot\left[\prod_{j=2}^{n-1}|[(B-A) \beta+A](j-2)+M|^{2}\right] \\
= & \frac{1}{((n-1) !)^{2}} \prod_{j=2}^{n}|[(B-A) \beta+A](j-2)+M|^{2} .
\end{aligned}
$$

Thus, we get

$$
\left|a_{n}\right| \leq \frac{1}{(n-1) !} \prod_{j=2}^{n}|[(B-A) \beta+A](j-2)+M|,
$$

which completes the proof of (5.23).

With a view to prove the assertion (5.24) of Theorem 6, suppose that $n>N+2$, where $N$ is given by (5.22). Then, retaining only the terms of the series on the right-hand side of (5.31) from $k=2$ to $k=N+2$, we have

$$
\begin{aligned}
& (n-2)^{2}\left|a_{n}\right|^{2} \leq(B-A)^{2} \beta^{2}(1-\alpha)^{2} \cos ^{2} \lambda \\
& \quad+\sum_{k=2}^{N+2}\left\{|[(B-A) \beta+A](k-1)+M|^{2}-(k-1)^{2}\right\}\left|a_{k}\right|^{2},
\end{aligned}
$$


where we have obviously dropped the non-negative termes from $k=N+3$ to $k=n-1$.

Now we substitute in (5.35) the upper bounds for

$$
\left|a_{2}\right|,\left|a_{3}\right|, \ldots,\left|a_{N+2}\right|
$$

given by (5.23), and the assertion (5.24) follows upon simplifying the resulting equation.

(b) Let the inequality (5.25) hold. Then it follows from (5.31) that

$$
(n-1)^{2}\left|a_{n}\right|^{2} \leq(B-A)^{2} \beta^{2}(1-\alpha)^{2} \cos ^{2} \lambda \text { for } n \geq 2,
$$

which evidently proves the assertion (5.26) of Theorem 6 .

The bound in (5.23) is sharp for the function $f(z)$ given by

$$
f(z)=z\{1-[(B-A) \beta+A] z\}^{-\frac{(B-A) \beta(1-\alpha) e^{-i \lambda} \cos \lambda}{[(B-A) \beta+A]}}, \beta \neq\left(\frac{A}{A-B}\right),
$$

and the bounds in (5.26) are sharp for the functions $f_{n}(z)$ given by

$$
f_{n}(z)= \begin{cases}z\left\{1-[(B-A) \beta+A] z^{n-1}\right\}^{\frac{-I(B-A) \beta(1-\alpha) e^{-i \lambda} \cos \lambda}{[(B-A) \beta+A](n-1)}} & \left(\beta \neq\left(\frac{A}{A-B}\right): n \geq 2\right) \\ z \exp \left[\frac{-A(1-\alpha) z^{n-1} e^{-i \lambda} \cos \lambda}{(n-1)}\right] & \left(\beta=\left(\frac{A}{A-B}\right) ; n \geq 2\right) .\end{cases}
$$

An immediate consequence of Theorem 6 may be stated as

Corollary 5. Let the function $f(z)$ defined by (1.1) be in the class $C^{\lambda}(\alpha, \beta, A, B)$. Then, under the hypotheses (5.21) and (5.22).

$$
\left|a_{n}\right| \leq \frac{1}{n !} \prod_{k=2}^{n} \mid[(B-A) \beta+A]\left[(k-2)+(B-A) \beta(1-\alpha) e^{-i \lambda} \cos \lambda \mid,\right.
$$

for $n=2,3, \ldots, N+2 ;$ and

$$
\begin{aligned}
& \left|a_{n}\right| \leq \frac{1}{(N+1) ! n(n-1)} \prod_{k=2}^{N+3} \mid[(B-A) \beta+A][(k-2) \\
& \quad+(B-A) \beta(1-\alpha) e^{-i \lambda} \cos \lambda \mid, \quad n>N+2 .
\end{aligned}
$$

(b) If, on the other hand, the condition (5.25) holds true, then

$$
\left|a_{n}\right| \leq \frac{(B-A) \beta(1-\alpha) \cos \lambda}{n(n-1)} \text { for } n \geq 2 \text {. }
$$

The estimates in (5.38) are sharp for the function $f(z)$ given by

$$
\begin{array}{r}
1+\frac{z f^{\prime \prime}(z)}{f^{\prime}(z)}=\frac{1-\left\{[(B-A) \beta+A]-(B-A) \beta(1-\alpha) e^{-i \lambda} \cos \lambda\right\} z}{1-[(B-A) \beta+A] z} \\
\left(\beta \neq\left(\frac{A}{A-B}\right)\right),
\end{array}
$$


while the estimates in (5.40) are sharp for the functions $f_{n}(z)$ given by

$$
f_{n}^{\prime}(z)= \begin{cases}\left\{1-[(B-A) \beta+A] z^{n-1}\right\}^{\frac{-(B-A) \beta(1-\alpha) e^{-i \lambda} \cos \lambda}{[(B-A) \beta+A](n-1)}} & \left(\beta \neq\left(\frac{A}{A-B}\right): n \geq 2\right) \\ \exp \left[\frac{-A(1-\alpha) z^{n-1} e^{-i \lambda} \cos \lambda}{(n-1)}\right] & \left(\beta=\left(\frac{A}{A-B}\right) ; n \geq 2\right) .\end{cases}
$$

\section{Radius of $\gamma$-Spiral and $\gamma$-Convex}

Let $S_{1}$ be the family of all normalized functions which are analytic and univalent in $U$. In [18], Libera introduced the concept of " $\gamma$-spiral radius" for the classes of univalent functions as follows:

Definition 3. If $f(z) \in S_{1}$ and $|\gamma|<\frac{\pi}{2}$, then the $\gamma$-spiral radius of $f(z)$ is

$$
\gamma-\text { s.r. }\{f(z)\}=\sup \left\{r: \operatorname{Re}\left(e^{i \gamma} \frac{z f^{\prime}(z)}{f(z)}\right)>0,|z|<r\right\} \text {. }
$$

Definition 4. If $F \subset S_{1}$ and $|\gamma|<\frac{\pi}{2}$, then the $\gamma$-spiral radius of $F^{\prime}$ is

$$
\gamma-\text { s.r. } F=\inf _{f \in F}[\gamma-\text { s.r. }\{f(z)\}]
$$

Also in [22], Mogra introduced the concept of " $\gamma$-convex radius" as follows:

Definition 5. If $f(z) \in S$, the class of analytic functions in $U$, and $|\gamma|<\frac{\pi}{2}$, then the $\gamma$-convex radius of $f(z)$ is

$$
\gamma-\text { c.r. }\{f(z)\}=\sup \left\{r: \operatorname{Re}\left\{e^{i \gamma}\left(1+\frac{z f^{\prime \prime}(z)}{f^{\prime}(z)}\right)\right\}>0,|z|<r\right\} .
$$

Definition 6. If $G \subset S$ and $|\gamma|<\frac{\pi}{2}$, then the $\gamma$-convex radius of $G$ is

$$
\gamma-\text { c.r.G }=\inf _{f \in G}[\gamma-\text { c.r. }\{f(z)\}]
$$
tion

Theorem 7. $\gamma$-s.r. $S^{\lambda}(\alpha, \beta, A, B)$, is the smallest positive root $r_{0}$ of the equa-

$$
\begin{aligned}
{[(B-A) \beta+A] } & \{(B-A) \beta(1-\alpha) \cos (\gamma-\lambda) \cos \lambda-[(B-A) \beta+A] \cos \gamma\} r^{2} \\
& -(B-A) \beta(1-\alpha) r \cos \lambda+\cos \gamma=0 .
\end{aligned}
$$

The result is sharp. 
Proof. Let $f(z) \in S^{\lambda}(\alpha, \beta, A, B)$. Then by using Lemma 1, we have

$$
\frac{z f^{\prime}(z)}{f(z)}=\frac{1+\left\{[(B-A) \beta+A]-(B-A) \beta(1-\alpha) e^{-i \lambda} \cos \lambda\right\} w(z)}{1+[(B-A) \beta+A] w(z)}
$$

where $w(z)$ satisfies the conditions $w(0)=0$ and $|w(z)|<\stackrel{1}{\text {. If }} B(z)=e^{i \gamma} \frac{z f^{\prime}(z)}{f(z)}$ and $|\gamma|<\frac{\pi}{2}$, then (6.6) may be written as

$$
w(z)=\frac{e^{i \gamma}-B(z)}{[(B-A) \beta+A] B(z)-e^{i \gamma}\left([(B-A) \beta+A]-(B-A) \beta(1-\alpha) e^{-i \lambda} \cos \lambda\right)}, z \in U
$$

Now by applying Schwarz's Lemma [15], it follows that $B(z)$ maps the disc $|z| \leq r$ onto a disc $|B(z)-\eta|<R$, where

$$
\eta=\frac{e^{i \gamma}\left\{1-[(B-A) \beta+A]\left([(B-A) \beta+A]-(B-A) \beta(1-\alpha) e^{-i \lambda} \cos \lambda\right) r^{2}\right\}}{1-[(B-A) \beta+A]^{2} r^{2}}
$$

and

$$
R=\frac{(B-A) \beta(1-\alpha) r \cos \lambda}{1-[(B-A) \beta+A]^{2} r^{2}}
$$

Hence $\operatorname{Re}\left(e^{i \gamma} \frac{z f^{\prime}(z)}{f(z)}\right) \geq 0$ if and only if

$$
\begin{gathered}
\operatorname{Re}\left\{\frac{e^{i \gamma}\left\{1-[(B-A) \beta+A]\left([(B-A) \beta+A]-(B-A) \beta(1-\alpha) e^{-i \lambda} \cos \lambda\right) r^{2}\right\}}{1-[(B-A) \beta+A]^{2} r^{2}}\right\} \\
\quad \geq \frac{(B-A) \beta(1-\alpha) r \cos \lambda}{1-[(B-A) \beta+A]^{2} r^{2}}
\end{gathered}
$$

which, on simplification, and with the aid of (6.2) concludes the proof of Theorem 7 .

The result is sharp for the function $f(z)$ given by

$$
f(z)= \begin{cases}z\{1-[(B-A) \beta+A] z\} \frac{-\left[(B-A) \beta(1-\alpha) e^{-i \lambda} \cos \lambda\right.}{[(B-A) \beta+A]}, & \beta \neq\left(\frac{A}{A-B}\right), \\ z \exp \left[-A(1-\alpha) z e^{-i \lambda} \cos \lambda\right], & \beta=\left(\frac{A}{A-B}\right),\end{cases}
$$

and

$$
\xi=\frac{r\left\{[(B-A) \beta+A] r-e^{i(\lambda-\gamma)}\right\}}{1-[(B-A) \beta+A] r e^{i(\lambda-\gamma)}} .
$$

Replacing $\left(\frac{z f^{\prime}(z)}{f(z)}\right)$ by $\left(1+\frac{z f^{\prime \prime}(z)}{f^{\prime}(z)}\right)$ in Theorem 7 and using Definition 6, we get the following result for the class $C^{\lambda}(\alpha, \beta, A, B)$. 
Corollary 6. $\gamma$-c.r. $C^{\lambda}(\alpha, \beta, A, B)$ is the smallest positive root $r_{0}$ of the equation (6.5). The result is sharp for the function $f(z)$ given by

$$
f^{\prime}(z)= \begin{cases}\{1-[(B-A) \beta+A] z\}^{\frac{-(B-A) \beta(1-\alpha) e^{-i \lambda} \cos \lambda}{[(B-A) \beta+A]}}, & \beta \neq\left(\frac{A}{A-B}\right), \\ \exp \left[-A(1-\alpha) z e^{-i \lambda} \cos \lambda\right], & \beta=\left(\frac{A}{A-B}\right),\end{cases}
$$

$\xi$ being defined (as before) by (6.12).

\section{Radius of Starlikeness and Convexity}

We first state and prove

Theorem 8. The sharp radius of starlikes of the class $S^{\lambda}(\alpha, \beta, A, B), \beta \neq$ $\left(\frac{A}{A-B}\right)$, is given by

$$
\begin{gathered}
r_{s}=2\left\{(B-A) \beta(1-\alpha) \cos \lambda+\left[(B-A)^{2} \beta^{2}(1-\alpha)^{2} \cos ^{2} \lambda-4[(B-A) \beta+A]^{2}\right.\right. \\
\left.\left.\cdot\left[\frac{(B-A) \beta(1-\alpha) \cos ^{2} \lambda}{[(B-A) \beta+A]}-1\right]\right]^{\frac{1}{2}}\right\}^{-1}
\end{gathered}
$$

The expression in (7.1) is real and finite only when $\beta \neq\left(\frac{A}{A-B}\right)$ and such that

$$
(B-A)^{2} \beta^{2}(1-\alpha)^{2} \cos ^{2} \lambda \geq 4[(B-A) \beta+A]^{2}\left[\frac{(B-A) \beta(1-\alpha) \cos ^{2} \lambda}{[(B-A) \beta+A]}-1\right] .
$$

Proof. From (4.5), we have

$$
\begin{aligned}
\operatorname{Re}\left\{\frac{z f^{\prime}(z)}{f(z)}\right\} & \\
& \geq \frac{1-(B-A) \beta(1-\alpha) r \cos \lambda+[(B-A) \beta+A]^{2}\left[\frac{(B-A) \beta(1-\alpha) \cos ^{2} \lambda}{[(B-A) \beta+A]}-1\right] r^{2}}{1-[(B-A) \beta+A]^{2} r^{2}},
\end{aligned}
$$

where $|z|=r$.

Thus $\operatorname{Re}\left\{\frac{z f^{\prime}(z)}{f(z)}\right\}>0$ for $|z|<r_{s}$, where $r_{s}$ is given by (7.1), provided that $\beta \neq\left(\frac{A}{A-B}\right)$ and the condition (7.2) is satisfied.

To show that (7.1) is sharp, we let $f(z)$ be given by (5.36) and put

$$
\xi=\frac{r\left\{[(B-A) \beta+A] r-e^{i \lambda}\right\}}{1-[(B-A) \beta+A] r e^{i \lambda}} .
$$


we thus obtain

$$
\frac{\xi f^{\prime}(\xi)}{f(\xi)}=\frac{1-(B-A) \beta(1-\alpha) r \cos \lambda-[(B-A) \beta+A]^{2}\left[\frac{(B-A) \beta(1-\alpha) \cos ^{2} \lambda}{[(B-A) \beta+A]}-1\right] r^{2}}{1-[(B-A) \beta+A]^{2} r^{2}}
$$

which obviously has a zero real part when $r$ is given by (7.1). This completes the proof of Theorem 8.

Making use of the relationship (1.6) between the classes $S^{\lambda}(\alpha, \beta, A, B)$ and $C^{\lambda}(\alpha, \beta, A, B)$, we can deduce the following consequence of Theorem 8 .

Corollary 7. The sharp radius of convexity of the class $C^{\lambda}(\alpha, \beta, A, B), \beta \neq$ $\left(\frac{A}{A-B}\right)$, is given by (7.1). The result is sharp for the function $f(z)$ given by (5.41), $\xi$ being defind (as before) by (7.3).

\section{References}

[1] O. P. Ahuja, "Certain generalization of the Robertson functions," Yokohama Math. J. 31 (1983), 5-11.

[2] M. K. Aouf, "Bounded p-valent Robertson functions of order $\alpha$," Indian J. Pure Appl. Math. 16 (1985), no. 7, 775-790.

[3] M. K. Aouf, "Bounded spiral-like functions with fixed second coefficient," Internat. J. Math. Math. Sci. 12 (1989), no. 1, 113-118.

[4] M. K. Aouf, "On coefficient bounds of a certain class of $p$-valent $\lambda$-spiral functions of order $\alpha$," Internat. J. Math. Math. Sci. 10 (1987), no. 2, 259-266.

[5] M. K. Aouf, "On a class of $p$-valent starlike functions of order $\alpha$," Internat. J. Math. Math. Sci. 10 (1987), no. 4, 733-744.

[6] M. K. Aouf, "On subclasses of starlike functions of order $\alpha$ and type $\beta$," Tamkang J. Math. 21 (1990), no. 1, 41-58.

[7] S. K. Bajpai and T. J. S. Mehrok, "On the coefficient structure and a growth theorem for the functions $f(z)$ for which $z f^{\prime}(z)$ is spirallike," Puibl. Inst. Math. (Boograd) (N.S.) 16 (30) (1973), 5-12.

[8] P. N. Chichra, "Regular functions $f(z)$ for which $z f^{\prime}(z)$ is $\alpha$-spirallike," Proc. Amer. Math. Soc. 49 (1975), 151-160.

[9] Dashrath and S. L. Shukla, "Coefficient estimates for subclass of spirallike functions," Indian J. Pure Appl. Math. 14 (1983), no. 4, 431-439.

[10] R. M. Goel, "A subclass of $\alpha$-spiral functions," Publ. Math. Debrecen 23 (1976), 79-84.

[11] R. M. Goel and B. S. Mehrok, "On the coefficients of a subclass of starlike functions," Indian J. Pure Appl. Math. 12 (1981), no. 5, 634-647.

[12] H. S. Gopalakrishna and P. G. Umarani, "Coefficient estimates for some classes of spiral-like functions," Indian J. Pure Appl. Math. 11 (1980), no. 8, 1011-1017.

[13] W. Janowski, "Some extremal problems of certain families of analytic functions," Ann. Polon. Math. 28 (1973), 297-326.

[14] O. P. Juneja and M. L. Mogra, "On starlike functions of order $\alpha$ and type $\beta$," Rev. Roumaine Math. Pures Appl. 23 (1978), 751-765.

[15] F. R. Keogh and E. P. Merkes, "A coefficient inequality for certain classes of analytic functions," Proc. Amer. Math. Soc. 20 (1969), 8-12.

[16] P. K. Kulshrestha, "Bounded Robertson functions," Rend. Mat. (6) 9 (1976), 137-150. 
[17] V. Kumar and S. L. Shukla, "Radii of spiral-likeness of certain analytic functions," Tamkang J. Math. 17 (1986), no. 4, 51-58.

[18] R. J. Libera, "Univalent $\alpha$-spiral functions," Canad. J. Math. 19 (1967), 449-456.

[19] R. J. Libera, and M. R. Ziegler, "Regular functions $f(z)$ for which $z f^{\prime}(z)$ is $\alpha$-spiral," Trans. Amer. Math. Soc. 166 (1972), 361-370.

[20] B. Maköwka, "On some subclasses of univalent functions," Zesz. Nauk. Polit. Lodzkiejnr 254, Z. 9 (1976), 71-76.

[21] M. L. Mogra, "On a class of starlike functions in the unit disc," I. J. Indian Math. Soc. 40 (1976), 159-161.

[22] M. L. Mogra, "Spirallike and Robertson functions with fixed secend coefficient," Riv. Mat. Univ. Parma (4) 9 (1983), 411-419.

[23] M. L. Mogra and O. P. Ahuja, "On spirallike functions of order $\alpha$ and type $\beta$," Yokohama Math. J. 29 (1981), 145-156.

[24] Z. Nehari, Conformal Mapping, McGrow-Hill Book Co., Inc., New york (1952).

[25] K. S. Padmanabhan, "On certain classes of starlike functions in the unit disc," J. Indian Math. Soc. 32 (1968), 89-103.

[26] D. A. Patil and N. K. Thakare, "On coefficient bounds of $p$-valent $\lambda$-spiral functions of order $\alpha$," Indian J. Pure Appl. Math. 10 (1979), 842-853.

[27] M. S. Robertson, "Univalent functions $f(z)$ for which $z f^{\prime}(z)$ is spirallike," Michigan Math. J. 16 (1969), 97-101.

[28] P. I. Sizuk, "Regular functions $f(z)$ for which $z f^{\prime}(z)$ is $\theta$-spiral shaped of order $\alpha$," Sibrisk. Mat. Z. 16 (1975), 1286-1290, 1371.

[29] L. Špaček, "Přispevĕk k teorii funkcki prostyčh," Časopis Pěst. Mat. Fys. 62 (1932), 12-19.

[30] D. J. Wright, "On a class of starlike functions," Compositio Math. 21 (1969), 122-124.

[31] J. Zamorski, "About the extremal spiral schlicht functions," Ann. Polen. Math. 9 (1962), 265-273.

Department of Mathematics, Faculty of Science, University of Mansoura, Mansoura, Egypt. 